\title{
Associated Factors to Immunization Timeliness and Completion in Preterm and Low Birth Weight Infants at Essos Hospital Center Yaounde
}

\section{Marie Kouya $^{1}$, Annie Carole Nga Motaze ${ }^{2}$, Jeannette Epée Ngoue ${ }^{3}$, Arsène Brunelle Sandie ${ }^{4}$, Paul Olivier Koki Ndombo², Anne Esther Njom Nlend1,2*}

\author{
${ }^{1}$ Higher Institute of Medical Technology, Nkolondom, University of Douala, Douala, Cameroon \\ ${ }^{2}$ Essos Hospital Centre, Unit of Neonatology, Yaoundé, Cameroon \\ ${ }^{3}$ Faculty of Medicine and Biomedical Sciences, University of Yaounde 1, Yaoundé, Cameroon \\ ${ }^{4}$ Pan African University of Nairobi, Nairobi, Kenya \\ Email: *anne.njom@gmail.com
}

How to cite this paper: Kouya, M., Motaze, A.C.N., Ngoue, J.E., Sandie, A.B., Ndombo, P.O.K. and Nlend, A.E.N. (2021) Associated Factors to Immunization Timeliness and Completion in Preterm and Low Birth Weight Infants at Essos Hospital Center Yaounde. Open Journal of Pediatrics, 11, 9-18.

https://doi.org/10.4236/ojped.2021.111002

Received: December 29, 2020

Accepted: February 6, 2021

Published: February 9, 2021

Copyright $\odot 2021$ by author(s) and Scientific Research Publishing Inc. This work is licensed under the Creative Commons Attribution International License (CC BY 4.0).

http://creativecommons.org/licenses/by/4.0/

(c) (i) Open Access

\begin{abstract}
Introduction: Vaccination is very often delayed in premature and low birth weight infants. However, timely vaccination is even more important in this population because of their increased susceptibility to infection. Objective: To assess immunization practice and factors associated with vaccine promptness and completeness in former preterm and low-birth-weight infants. Methods: We conducted a retrospective analytical cross-sectional study (January 2017 to February 2019). Main Measurement: Promptness and completeness at each contact, Statistical analysis was performed using $\mathrm{R}$ software version 3.6.2, logistic regression was used to estimate the Odds Ratio (OR) and their 95\% Confidence Interval (CI). Results: We recruited 310 children aged 12 to 36 months born before 37 weeks with low birth weight, 163 (52.6\%) of whom were female. Two hundred and fifty-three had received the vaccines at the indicated age, with promptness rate of $81.6 \%$, and $97.7 \%$ had completed routine immunization at 9 months. The mean age at vaccination initiation was 6 days \pm 11 and the mean weight at vaccination initiation was $2233 \mathrm{~g} \pm 494$. High prematurity and very low birth weight were associated with a high rate of vaccine delay: 61.5\% [OR: 15.56; (CI: $3.22-118.52 ; \mathrm{p}=0.002$ )] and 66.7\% [OR: 19.19; (CI: 4.67 - 92.52; $\mathrm{p}<0.001$ )] respectively. Distance $>5 \mathrm{~km}$ with EHC [OR: 3.48; (CI: $1.68-7.47 ; \mathrm{p}=0.001)$ ] was associated with poor vaccination. Women in common-law unions had the lowest vaccine readiness rate $(60.6 \%)$, (OR: 3.36; CI: $1.006-10.70 ; \mathrm{p}=0.038$ ). The frequency of occurrence of post
\end{abstract}


immunization adverse events was $24.5 \%$, with fever type in $94.7 \%$. Conclusion: Almost all premature and/or low-birth-weight children hospitalized at Essos Hospital Center had completed routine immunization at 9 months, and the majority had received the vaccines in a timely manner. Similar study is needed in rural area.

\section{Keywords}

Prematurity, Low Birth Weight, Immunization, Timeliness, Completion

\section{Introduction}

One of the goals of the Global Plan of Action for Immunization (GPAI) 20112020 is to improve health by extending the full benefits of immunization to all people in an equitable manner [1]. Immunization is the process of making a person immune to an infectious disease, usually by administering a vaccine [2]. Vaccination has been shown to control and eliminate potentially fatal infectious diseases and it is estimated that more than 2 - 3 million deaths are prevented worldwide each year [2].

In 1988, Roper et al. noted a delay in immunization of children weighing less than $2000 \mathrm{~g}$ despite the recommendation that preterm and low birth weight children should receive vaccines as early as three months after birth [3]. As of 2011, the Advisory Committee on Immunization Practice (ACIP) of the Center for Disease Control (CDC) recommends that these children be vaccinated according to the same schedule and chronological age as children born at term, with the exception of hepatitis B vaccine at birth [4]. Despite the proven benefits of immunization and the numerous vaccine safety studies conducted in recent years, immunization is very often delayed in preterm, low-birth-weight infants [5] [6] [7] [8].

However, timely vaccination is even more important in low-birth-weight infants, due to an immature immune system and sub-optimal maternal antibody transfer that increases susceptibility to infection [9]. In Cameroon, there are no data on the implementation of the new recommendations on immunization of preterm and low birth weight children, which is why we set ourselves the goal of evaluating vaccine practice and factors associated with vaccine readiness and completeness in former preterm and low birth weight children.

\section{Materials and Methods}

Our study was conducted in the neonatology department of the Essos Hospital Center (EHC) in Yaounde. We included children from 12 to 36 months born before 37 weeks of amenorrhea (WA) and/or with a birth weight below $2500 \mathrm{~g}$. Children who died before the age of 9 months and those whose parent could not be contacted were excluded. We consulted the hospitalization registers of the 
EHC neonatology department and recorded the telephone contacts of the parents of children meeting our inclusion criteria born between January 2017 and February 2019. We contacted these parents and made our interviews by phone, as the health context due to Covid 19, did not allow us to go and meet them. A verbal informed consent was obtained before the beginning of the interview.

Data were collected from the mothers' statements and vaccination records. The variables studied were the basic vaccines, age-appropriate vaccines, and the socio-demographic characteristics of the mother and child. The data were analyzed using $\mathrm{R}$ software version 3.6.2. In bivariate analysis, cross-tabulations were determined, the Chi 2 test was used to perform the test of independence between the dependent variable (Promptness + Completeness) and the independent variables, and the Fisher's exact test was used alternatively when the conditions for applicability of the Chi 2 test were not met. In multivariate analysis, logistic regression was used to estimate the Odds Ratio (OR) and its 95\% Confidence Interval (CI) to look for the factors of vaccine promptness and completeness. The significance threshold was set at a value of $\mathrm{p}<0.05$.

\section{Ethical Considerations}

Ethical clearance for the study was obtained from the Institutional review board (IRB) of the Essos Hospital Centre under the reference number 2020/05/CE-CHE and the Institutional Ethics Committee of the University of Douala; in addition, the Hospital Directorate provided an administrative authorization.

\section{Results}

\subsection{General Characteristics}

We identified a total of 384 children who met our inclusion criteria. Seventy-four were excluded, including 64 unavailable phone numbers, 6 infant deaths before the age of 9 months and 4 refusals to participate (see Figure 1). We included $310(80.73 \%)$ children in our study, $36.5 \%$ of who were between 12 and 24 months of age and $63.5 \%$ between 24 and 36 months of age. The predominant gender was female, $52.6 \%$ with a sex ratio of 1.10 . More than $3 / 4$ of our population or $78.4 \%$ had average prematurity, $12.6 \%$ had a gestational age of $<32$ weeks. Those with a birth weight $<1500 \mathrm{~g}$ represented $9.7 \%$ and $58.7 \%$ had a weight included between 1500 and 2499 g. Regarding the socio-demographic characteristics of the mothers, the most represented age group was 26 - 40 years, $62.9 \%$ were married and $73.9 \%$ had a higher education level (Table 1).

\subsection{Immunization Coverage}

For Expanded Program on Immunization (EPI) vaccines, 253 children had received their vaccines at the indicated age, a promptness rate of $81.6 \%$. Almost all (97.7\%) had completed routine immunization at 9 months of age, and 167 (53.9\%) had received at least one dose of non-EPI vaccine. Two hundred and seventy-four (88.4\%) children had received the first vaccine within 7 days of birth. We recov- 
ered a promptness rate of $88.4 \%$ for BCG and OPV 0 vaccines received at the first contact (at birth), and a rate of $91.3 \%$ for the Diphtheria-Tetanus-Pertussis-Hepatitis B-Haemophilus influenza type b vaccine (DTP-HepB/Hib) + OPV+ Pneumococcal Conjugated Vaccine (PCV) + Rotavirus vaccine at 6 weeks, $90.3 \%$ for $\mathrm{DTP}-\mathrm{HepB} / \mathrm{Hib}+\mathrm{OPV}+\mathrm{PCV}+$ Rotavirus vaccine at 10 weeks, $98.4 \%$ for DTP-HepB/Hib + OPV + PCV + Inactivated Poliomyelitis Vaccine (IPV) at 14 weeks and $98.1 \%$ for the yellow fever and measles. Vitamin A supplementation at 6 months had the highest promptness rate (98.4\%).The completion rate at first contact was $100 \%$ and this rate decreased for subsequent contacts but was still satisfactory ( $>90 \%$ ) (See Figure 2).

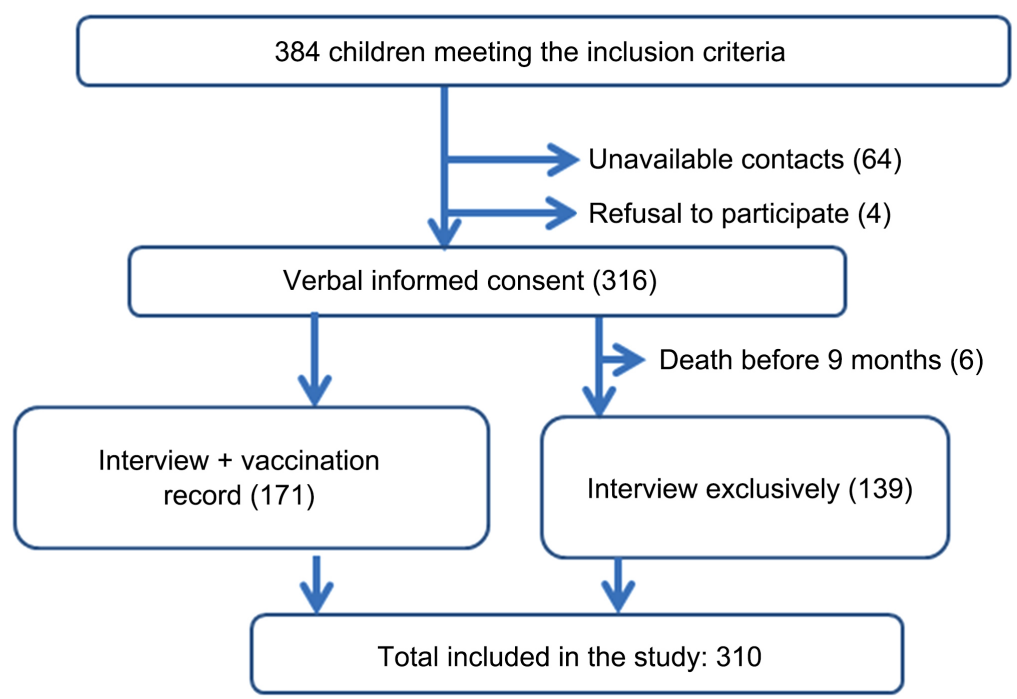

Figure 1. Flow diagram of the study population.

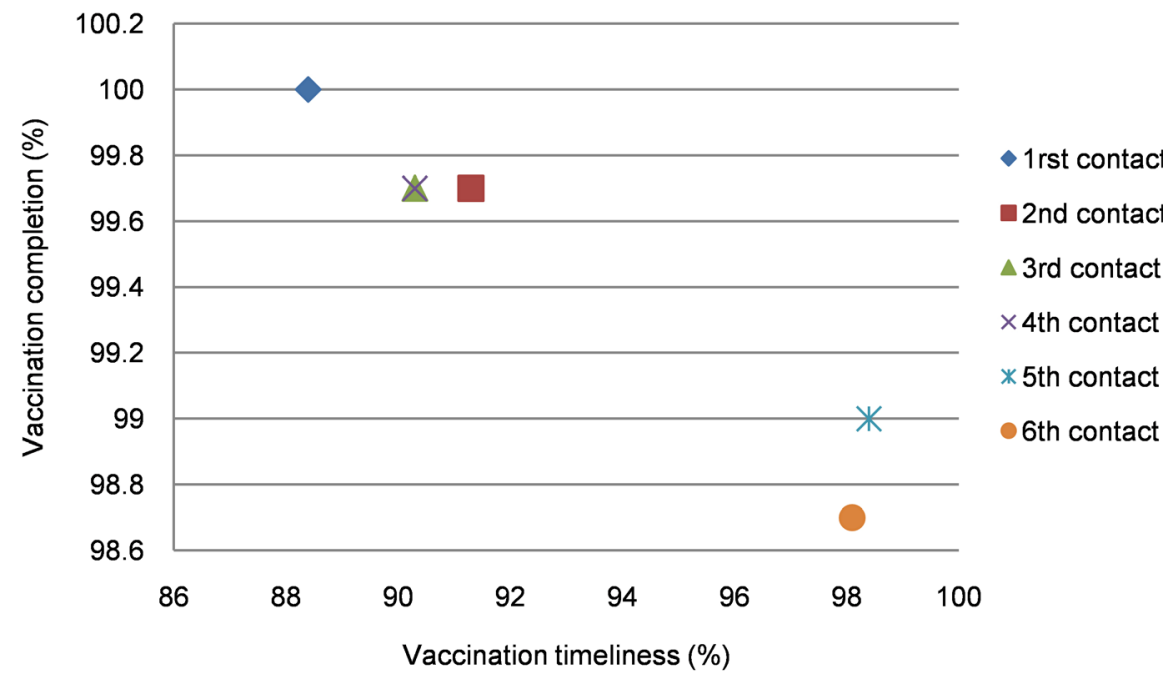

Figure 2. Timeliness and completion of vaccination for each vaccine contact. 1rst contact: at birth (BCG + OPV 0); 2nd contact: 6 weeks (DTP-HepB/Hib + OPV + PCV + Rotarix); 3rd contact: 10 weeks (DTP-HepB/Hib + OPV + PCV + Rotarix); 4th contact: 14 weeks (DTP-HepB/Hib + OPV + PCV + IPV); 5th contact: 6 months (Vitamin A supplementation); 6th contact: 9 months (measles + yellow fever vaccine). 


\subsection{Factors Influencing Vaccine Readiness}

In multivariate analysis, children with extreme prematurity were nearly 16 times less likely to be vaccinated [OR: 15.56 (CI: $3.22-118.52$ ), $\mathrm{p}=0.002$ ] and those with very low birth weight were 19 times less likely to be vaccinated [OR: 19.19 (CI: 4.67 - 95.52), $\mathrm{p}<0.001]$. Children whose mothers were in common-law unions had the lowest vaccine readiness rate of $60.6 \%$ [OR: 3.36 (CI: $1.06-10.70), p$ $=0.038$ ] and mothers living more than $5 \mathrm{~km}$ from HEC were three times less likely to vaccinate their children [OR: 3.48 (CI: $1.68-7.47$ ), $\mathrm{p}=0.001$ ], as shown in Table 2. Of all children vaccinated, 76 (24.5\%) had MAPI, including fever (94.7\%), rash (2.65\%), and injection site abscess $(2.65 \%)$. We did not find a significant association with the maternal age $(\mathrm{p}=0.981)$, the maternal education ( $\mathrm{p}$ $=0.819)$, the maternal occupation $(\mathrm{p}=0.147)$, the religion $(\mathrm{p}=0.770)$, the occurrence of adverse events $(p=0.229)$, the route of delivery $(p=0.189)$ and maternal interest in vaccination $(\mathrm{p}=1)$.

Table 1. Socio-demographic characteristics of the mothers.

\begin{tabular}{|c|c|c|}
\hline & Frequency & Percentage (\%) \\
\hline \multicolumn{3}{|l|}{ Age of the mother: } \\
\hline - $15-25$ & 31 & 10 \\
\hline - $26-40$ & 265 & 85.6 \\
\hline - $41-50$ & 14 & 4.5 \\
\hline \multicolumn{3}{|l|}{ Marital status: } \\
\hline - Single & 82 & 26.5 \\
\hline - Divorced & 1 & 0.3 \\
\hline - Married & 195 & 62.9 \\
\hline - Common-law union & 32 & 10.3 \\
\hline \multicolumn{3}{|l|}{ Education level: } \\
\hline - Primary & 1 & 0.3 \\
\hline - Secondary & 80 & 25.8 \\
\hline - Higher & 229 & 73.9 \\
\hline \multicolumn{3}{|l|}{ Profession: } \\
\hline - Housewife & 56 & 18.1 \\
\hline - Student & 38 & 12.3 \\
\hline - Informal & 48 & 15.5 \\
\hline - Private & 96 & 31.0 \\
\hline - Public & 72 & 23.2 \\
\hline \multicolumn{3}{|l|}{ Religion: } \\
\hline - Christian & 294 & 94.8 \\
\hline - Muslim & 16 & 5.2 \\
\hline
\end{tabular}


Table 2. Associated factors to vaccine delay: multivariate analysis.

\begin{tabular}{|c|c|c|c|c|c|c|}
\hline \multicolumn{2}{|c|}{ Vaccination timeliness } & \multirow{2}{*}{$\begin{array}{c}\text { Untimely N(\%) } \\
2(7.1)\end{array}$} & \multirow{2}{*}{$\begin{array}{c}\text { Timely N(\%) } \\
26(92.9)\end{array}$} & \multirow{2}{*}{$\begin{array}{l}\text { Non adjusted OR } \\
(95 \% \mathrm{CI}, \mathrm{p}) \\
-\end{array}$} & \multirow{2}{*}{$\begin{array}{c}\text { AdjustedOR } \\
(95 \% \mathrm{CI})\end{array}$} & \multirow[t]{2}{*}{ p-value } \\
\hline & Normal & & & & & \\
\hline Gestational Age & Moderateprematurity & $31(12.8)$ & $212(87.2)$ & $\begin{array}{c}1.90(0.53-12.16 \\
p=0.397)\end{array}$ & $2.79(0.73-18.44)$ & $\mathrm{p}=0.191$ \\
\hline & High prematurity & $24(61.5)$ & $15(38.5)$ & $\begin{array}{c}20.80(5.20-141.28 \\
\mathrm{p}<0.001)\end{array}$ & $15.56(3.22-118.52)$ & $\mathrm{p}=0.002$ \\
\hline \multirow{3}{*}{ Birth weight } & $>=2500 \mathrm{~g}$ & $4(4.1)$ & $94(95.9)$ & - & - & \\
\hline & LBW & $33(18.1)$ & 149 (81.9) & $\begin{array}{c}5.20(1.99-17.87 \\
\mathrm{p}=\mathbf{0 . 0 0 2 )}\end{array}$ & $5.26(1.90-18.74)$ & $\mathrm{p}=0.004$ \\
\hline & VLBW & $20(66.7)$ & $10(33.3)$ & $\begin{array}{c}47.00(14.66-188.70 \\
\mathrm{p}<0.001)\end{array}$ & $19.19(4.67-92.52)$ & $\mathrm{p}<0.001$ \\
\hline \multirow{3}{*}{ Marital status } & Single & $16(19.5)$ & $66(80.5)$ & - & - & \\
\hline & Cohabitation & $13(39.4)$ & $20(60.6)$ & $\begin{array}{c}2.68(1.10-6.55 \\
\quad \mathrm{p}=0.029)\end{array}$ & $3.36(1.06-10.70)$ & $\mathrm{p}=0.038$ \\
\hline & Married & $28(14.4)$ & $167(85.6)$ & $\begin{array}{c}0.69(0.35-1.39 \\
p=0.286)\end{array}$ & $0.66(0.29-1.49)$ & $\mathrm{p}=0.309$ \\
\hline \multirow[b]{2}{*}{$\begin{array}{c}\text { Distance from Esso } \\
\text { Hospital Center }\end{array}$} & Lessthan $5 \mathrm{~km}$ & $26(13.3)$ & $170(86.7)$ & - & - & \\
\hline & More than $5 \mathrm{~km}$ & $31(27.2)$ & $83(72.8)$ & $\begin{array}{c}2.44(1.36-4.40 \\
\quad p=0.003)\end{array}$ & $3.48(1.68-7.47)$ & $\mathrm{p}=0.001$ \\
\hline
\end{tabular}

LBW: low birth weight; VLBW: Very Low Birth Weight; OR: Odd Ratio.

\section{Discussion}

Our retrospective study showed that almost all premature and/or low birth weight children hospitalized in the EHC neonatal department (97.7\%) had completed the vaccination at 9 months. BCG and OPV 0 are routinely administered to preterm and low birth weight infants hospitalized in the EHC neonatal unit, which may explain the $100 \%$ completion rate for BCG and OPV 0 in our study. This confirms the results of the 2011 study by Denizot et al. in France, who found that the first vaccination before hospital discharge was associated with a better vaccination coverage rate [10].

We found that $81.6 \%$ of children were vaccinated at the age indicated in the EPI schedule, a figure almost twice as high as that found by Nakatudde et al. in 2019 in Uganda, which was 42.6\%. Overall promptness in our study decreased with low gestational age (38.5\% for those born before $32 \mathrm{WA})$ and with low birth weight $(33.3 \%$ for those with birth weight below $1500 \mathrm{~g})$. This finding was also made in a recent study in the Netherlands by E. Rouers et al. who found a $60.5 \%$ promptness that dropped to $37 \%$ in children with gestational age $<28$ WA [11].

We observe that children born with low birth weight and gestational age far from term were more likely to have delayed vaccination. We found a very signif- 
icant association between high prematurity, very low birth weight, low birth weight and not prompt vaccination. This confirms the finding of Maureen O'Leary et al. in Ghana with low birth weight as a risk factor for poor immunization even for children born in health facilities [12].

Zero-dose oral polio vaccine (OPV 0) should be given at birth or as soon as possible thereafter to maximize seroconversion rates with subsequent doses and induce mucosal protection [13]. In our study, we found $88.4 \%$ promptness for BCG and OPV 0 valences. Only $11.6 \%$ had received these vaccines at more than 7 days, which is lower than the figures from the study on reasons and determinants of delay of BCG vaccination in a community in sub-Saharan Africa by Bolajoko O Olusanya, in which $65.4 \%$ were vaccinated at 2 weeks, and $24.5 \%$ at 6 weeks [14]. Nakatudde et al. found that in 2019 in Uganda, BCG was 92\% prompt and OPV, 45.4\% prompt, both received at birth [15]. This disparity was due to stock-outs of OPV at Mulago Hospital in Uganda during the study period [15]. In our study, we found similar readiness for BCG and OPV 0 , indicating the availability of these vaccines in our health facility.

For diphtheria, tetanus, pertussis, polio, rotavirus, pneumococcal, and measles vaccines, we found $>90 \%$ readiness for DTP-HepB/Hib, (at 6, 10 and 14 weeks), Rotarix (at 6 and 10 weeks), measles + yellow fever vaccine (at 9 months). These results are more than three times better than those of Pinquier et al. who found only 28 percent of preterm infants meeting the French recommendations for DTP-Hib, MMR, and HepB at age 2 years [16].

In this setting, children born with low birth weight and those born at an early age began vaccination later than children born at term and/or birth weight $\geq 2500 \mathrm{~g}$. The mean age of vaccination initiation was 6 days \pm 11 . This age is similar to that found in Ghana by Maureen O Leary et al. [12]. We found a significant association between birth weight and vaccination initiation $(\mathrm{p}<0.001)$ with a mean vaccination initiation weight of $2233 \mathrm{~g} \pm 494$.

On the opposite, we did not find a significant association with maternal age, maternal education, occupation, religion, route of delivery, occurrence of adverse events, and maternal interest in vaccination. Our findings may contradict those of Ravi Prakash et al., who identified Muslim religion, maternal age $<20$ years as a factor associated with low vaccine completion rates among preterm infants in India [17]. They also found that maternal education ( $\geq 12$ years of schooling) was a factor in satisfactory completion rates [17].

Distance from home to EHC greater than $5 \mathrm{~km}$ was associated with a high rate of no promptness compared with a distance of less than $5 \mathrm{~km}$ [OR: 3.48 (CI: 1.68 - 7.47, $\mathrm{p}=0.001)$ ]. This result is concordant with data posted by Maureen O'Leary et al. in 2017 in Ghana, who found that children living more than $5 \mathrm{~km}$ from the health facility were 1.37 times unvaccinated compared to those living 1 $\mathrm{km}$ from the health facility ( $\mathrm{p} \leq 0.0001)$ [12]. This implies the need to educate mothers of preterm and low birth weight infants about the importance of continuing immunization at the health facility closest to home in case of difficulty in 
reaching the hospital where vaccination was started at birth.

Concerns about the safety of vaccines in preterm and low birth weight infants are often the reason for delayed vaccination, despite the fact that the frequency of adverse events appears to be comparable between preterm and term infants [18]. Studies have shown that children with prior cardiorespiratory problems are at greater risk of developing these adverse reactions [19] [20]. In our study, $24.5 \%$ of children had an adverse reaction after vaccination, made of fever and local reactions (5.3\%).

Limitations of the study: The unavailability of some children's immunization records during the interview, involving the memory of the mother being interviewed. In these cases, it is possible that some information may not have been collected with certainty. Therefore, there is a risk of recall bias in this study because parents may not recall certain information accurately. Access to photographs of the immunization records of many mothers after interviewing, and information in the hospitalization records of the neonatal unit helped to minimize this problem, but the unverified records could influence vaccine completion and readiness rates. We did not investigate the knowledge of new recommendations on vaccination of preterm and low birth weight infants by medical staff as a factor influencing vaccination practice in our study population. Nevertheless, the information collected from the mothers interviewed allowed us to identify the main factors influencing vaccine readiness and completion in the preterm and low birth weight population, and analysis of these factors is necessary to improve vaccine coverage in this vulnerable population.

\section{Conclusion}

Almost all premature and/or low birth weight children had completed routine immunization by 9 months, and the majority had received the vaccines at the indicated age. Very low birth weight, extreme prematurity, and distance $>5 \mathrm{~km}$ from home to EHC were predictive factors for delayed immunization. It is recommended that medical staff should ensure that all newborns are vaccinated regardless of birth weight and term. In addition, ongoing training should be provided to immunization staff regarding the vaccination of preterm and low birth weight infants.

\section{Acknowledgements}

We want to express our gratitude to all the mothers and parents for their willingness to participate in this study, as well all the staff of the neonatal unit and the immunization service.

\section{Author's Contributions}

KM and AENN designed the study, KM, ACNM and ENJ ensured data collection and curation, $\mathrm{ABS}$ analyzed the data. $\mathrm{KM}$ and AENN wrote the manuscript. POKN and BS reviewed the manuscript. All authors approved the final version. 


\section{Conflicts of Interest}

The authors declare no conflicts of interest regarding the publication of this paper.

\section{References}

[1] OMS (2013) Plan d'action mondial pour les vaccins 2011-2020.

[2] OMS (2018) Vaccination [Internet]. WHO, Geneva. https://www.who.int/topics/immunization/fr

[3] Roper, J. and Day, S. (1988) Uptake of Immunisations in Low Birthweight Infants. Archives of Disease in Childhood, 63, 518-521. https://doi.org/10.1136/adc.63.5.518

[4] Centers for Disease Control and Prevention (2011) General Recommendations on Immunization Recommendations of the Advisory Committee. MMWR Recommendations and Reports, 60, 25-26.

[5] Omeñaca, F., Vázquez, L., Garcia-Corbeira, P., et al. (2018) Immunization of Preterm Infants with GSK's Hexavalent Combined Diphtheria-Tetanus-Acellular PertussisHepatitis B-Inactivated Poliovirus-Haemophilus Influenzae Type b Conjugate Vaccine: A Review of Safety and Immunogenicity. Vaccine, 36, 986-996. https://doi.org/10.1016/j.vaccine.2018.01.005

[6] Laforgia, N., Di Mauro, A., Bianchi, F.P., et al. (2018) Are Pre-Terms Born Timely and Right Immunized? Results of an Italian Cohort Study. Human Vaccines \& Immunotherapeutics, 14, 1398-1402. https://doi.org/10.1080/21645515.2018.1428509

[7] Badurdeen, S., Marshall, A., Daish, H., et al. (2019) Safety and Immunogenicity of Early Bacillus Calmette-Guérin Vaccination in Infants Who Are Preterm and/or Have Low Birth Weights: A Systematic Review and Meta-Analysis. JAMA Pediatrics, 173, 75-85. https://doi.org/10.1001/jamapediatrics.2018.4038

[8] Duan, K., Guo, J. and Lei, P. (2017) Safety and Immunogenicity of Pneumococcal Conjugate Vaccine in Preterm Infants: A Meta-Analysis. The Indian Journal of Pediatrics, 84, 101-110. https://doi.org/10.1007/s12098-016-2248-2

[9] Sisson, H. (2014) Vaccinating Preterm Infants: Why the Delay? Infant, 10, 84-86.

[10] Denizot, S., Fleury, J., Caillaux, G., et al. (2011) Hospital Initiation of a Vaccinal Schedule Improves the Long-Term Vaccinal Coverage of Ex-Preterm Children. Vaccine, 29, 382-386. https://doi.org/10.1016/j.vaccine.2010.11.006

[11] Rouers, E.D.M., Berbers, G.A.M., van Dongen, J.A.P., et al. (2019) Timeliness of Immunisations in Preterm Infants in the Netherlands. Vaccine, 37, 5862-5867. https://doi.org/10.1016/j.vaccine.2019.08.006

[12] O'Leary, M., Edmond, K., Floyd, S., Hurt, L., et al. (2017) Neonatal Vaccination of Low Birthweight Infants in Ghana. Archives of Disease in Childhood, 102, 145-151. https://doi.org/10.1136/archdischild-2016-311227

[13] OMS (2019) Tableau 1: Vaccination systématique recommandée-Résumé des notes d'information de l'OMS.

[14] Olusanya, B.O. (2010) Pattern and Determinants of BCG Immunisation Delays in a Sub-Saharan African Community. Health Research Policy and Systems, 8, 1-10. https://doi.org/10.1186/1478-4505-8-1

[15] Nakatudde, I., Rujumba, J., Namiiro, F., et al. (2019) Vaccination Timeliness and Associated Factors among Preterm Infants at a Tertiary Hospital in Uganda. PLoS ONE, 14, e0221902. https://dx.plos.org/10.1371/journal.pone.0221902 
https://doi.org/10.1371/journal.pone.0221902

[16] Pinquier, D., Adde-Michela, C., Ploin, D., et al. (2009) Couverture vaccinale des grands prématurés à 6 mois et à 2 ans: Etude pilote. Archives de Pédiatrie, 16, 1533-1539. https://doi.org/10.1016/j.arcped.2009.09.009

[17] Upadhyay, R.P., Chowdhury, R., Mazumder, S., et al. (2017) Immunization Practices in Low Birth Weight Infants from Rural Haryana, India: Findings from Secondary Data Analysis. Journal of Global Health, 7, Article ID: 020415. https://doi.org/10.7189/jogh.07.020415

[18] Sioriki, A., Gkentzi, D., Papadimitriou, E., et al. (2020) Vaccinations in Infants Born Preterm: An Update. Current Pediatric Reviews, 16, 148-155.

https://doi.org/10.2174/1573396316666200116094459

[19] Bonhoeffer, J., Siegrist, C.A. and Heath, P.T. (2006) Immunisation of Premature Infants. Archives of Disease in Childhood, 91, 929-935. https://doi.org/10.1136/adc.2005.086306

[20] Esposito, S., Serra, D., Gualtieri, L., et al. (2009) Vaccines and Preterm Neonates: Why, When, and with What. Early Human Development, 85, S43-S45.

https://doi.org/10.1016/j.earlhumdev.2009.08.011 